\title{
Bayesian Filtering for Bluetooth RSS-based Indoor Tracking
}

\author{
Bao Zhenshan, Wang Lingze, Zhang Wenbo \\ College of Computer Science, Beijing University of Technology \\ Beijing, China \\ baozhenshan@bjut.edu.cn
}

\begin{abstract}
With the technical advances of wireless sensor networking and smart mobile device, the demand for position information of pedestrians (especially in the indoor environment) has increased remarkably. In this paper, we proposed an indoor localization approach based on received signal strength (RSS) and Bayesian filter. In the following sections, we describe our virtual modeling method of environment and the way we take object's movement sequences as history conditions in Bayesian filter. The experiment results show that our solution provides accurate tracking results (within 80 centimeters for moving object). The contribution of this research is that it provides a general implementation utilizing Bayesian filter which is able to estimate location precisely with off-the-shelf hardware. And Bluetooth Low Energy (BLE) is employed which reduces power consumption considerably. Meanwhile the accuracy is sufficient for pedestrian tracking in real application scenarios where BLE devices can be easily deployed.
\end{abstract}

Keywords-indoor localization, pedestrian tracking, bluetooth low energy, received signal strength, Bayes filter.

\section{INTRODUCTION}

As the basic context of wireless sensor network, location is one of the key information. From radar and sonar technology to GPS, localization technology has always been developed for general or military purposes. Since the need of indoor localization has been proposed, the situation has changed significantly. Now wireless sensors and portable devices are cheap and easy to get, which makes indoor location based service (LBS) possible and affordable. Just like the variety of LBS applications, people need various positioning means along with GPS. In the first decade of research, advances of technology have inspired numerous solutions for almost all kinds of wireless communication technology, such as RADAR [1] for IEEE802.11, LANDMARC [2] for RFID and Cricket [3] for acoustic signal.

In that first decade, comparison of different technologies was the main topic. Numerous solutions were discussed such as WiFi, RFID, Bluetooth, infra-red, ultrasonic, Zigbee. After that, signal processing engineering became the hotspot of indoor localization research. The most widely discussed is filter technique which is used for the improvement of accuracy. For the tracking problem, these methods for static targets are not adequate. That is because location estimation is made by measurements which are limited by the sampling frequency of sensors. No signal is measured during sampling intervals.
Simply connecting static points will distort the real trajectory. One solution for this problem is filter technique. Filter technique is not only can be used in signal measurements, but also on general position estimations.

In this paper we propose a Bluetooth indoor pedestrian tracking system which employs RSS as scene profiling basis. During the tracking process, our system keeps iterating a recursion of prediction and update in which Bayes filter [4] is the mathematical fundamental. Other than the classic procedure of filtering the measurement data only, our filter integrates context information and history record as conditions for the estimation. Our evaluation shows that our combination of current measurements with history records increases both the accuracy and efficiency. The rest of this paper is organized as follows: Section II overviews fundamental and essential related works. Then Section III describes detailed implementation. After that in Section IV we present an evaluation of the proposed algorithm by comparing it with existing RSS-based fingerprint approaches. Finally, Section VI summarizes the main conclusions and gives directions for future work.

\section{RELATED WORKS}

In this section, we summarize recent work of indoor localization and discuss the limitation of current state of art. Our classification of recent works is based on signal types, signal metrics and signal processing methods.

\section{A. Signal Types}

Optical signals, acoustic signals and radio signals are the most commonly used physical quantities. Most systems using optical signals are based on infrared rays while some others are based on pattern recognition. Active Badge [5] is an early implementation based on infrared. This system detects infrared signals from special badges carrying by target. Its successor Active Bat [6] turns to use acoustic signals for localization. Active Bat also requires users carry a sensor tag which transmits and receives ultrasound signals. Besides Active Bat, MIT Cricket is another ultrasound based localization method. Better than Active Bat, it has decentralized administration, network heterogeneity, and low cost.

\section{B. Signal Metrics}

To estimate the position of target, various context information can be taken advantage of. The most commonly talked about include: AOA [7] (angle of arrival), TOA [8] (time 
of arrival), TDOA [9] (time difference of arrival) and RSS (received signal strength). The former three utilize peculiarities of various signals to calculate the absolute location based on the absolute coordinates of sensors and relative position of target. As long as the obstacles are absent and the signal interferences are slight, their granularity can achieve up to 1 meter or better easily. However, these three kinds of approaches require dedicated infrastructure which is not the necessity of RSS measurements. Our system implementation is based on the Bluetooth Low Energy (BLE) which is not easy to measure the difference of distance, time or angle accurately. Thus our implementation follows the common RSS-based indoor localization approach.

\section{Signal Processing Methods}

As mentioned above, RSS-based localization approach usually does not depend on triangulation. Besides triangulation there is another method which is finger-print approach. In an indoor environment, multi sensors would be deployed. Each sensor could supply several unique signal values such as signal strength. These unique values construct a signature (also called finger-print) for each position. The process of capturing and recording these finger-prints called training phase (or scene profiling). After the work of training phase is done, clients need localization service could upload the finger-print of its current position which will be compared with prior history record. Most RSS-based indoor localization approach is the use of the kNearest Neighbor algorithm $(k-N N)$ [10]. By this means, RSS map is constructed by averaging separately the RSS values received from each wireless access point (AP). It records signature vectors for all the positions during the training phase. During runtime the algorithm calculates the Euclidean distance between current signature and history records then sorts the $\mathrm{k}$ closest neighbors in increasing order. The final estimated position is given by computing the centroid of these $\mathrm{k}$ closest neighbors. In section $\mathrm{V}$ this algorithm is used in the first step of the recursion.

\section{DESIGN AND IMPLEMENTAION}

In this section we will describe our design and implementation in detail. First of all, we need to introduce our underlying communication protocol and our devices briefly. Then we will present our modeling method for the indoor environment where our system is going to be deployed. Finally, our Bayes filter implementation is given.

\section{A. Beacon Device and Central Console}

As mentioned in section II, we use radio signals other than optical or acoustic signals to locate the target. After weighing the balance of cost and precision, we decide to employ a recently presented wireless networking protocol: Bluetooth low energy (BLE), alias Bluetooth Smart. BLE is the Version 4.0 of Bluetooth protocol. Although it operates in the same spectrum range (the $2.400 \mathrm{GHz}-2.4835 \mathrm{GHz}$ ISM band) as Classic Bluetooth technology, it uses a different set of channels. And data are transmitted using Gaussian frequency shift modulation. Along with frequency hopping, BLE is able to counteract narrowband interference problems. Furthermore, just as its name implies, Bluetooth Low Energy makes the cost of deployment and maintenance significantly decreased. In our experiment, the price for each beacon device is lower than 10 dollars and one button battery can support the beacon for more than 6 months. This means that we can increase redundancy to avoid individual device failure or temporary invalid.

In our experiment a regular smart phone is used to be the central console. Our central console scans surrounding circumstances every 50 milliseconds.

\section{B. Modeling of the indoor environment}

For ease of calculation and measurement, we model our experimental indoor environment as several two-dimensional grids and make an assumption that target could only move across the grid discretely.

We plant our Bluetooth beacons at specific points. And we measure the received signal-strength of our target with all planted beacons. For each beacon we can capture a measurement of RSS every 500 milliseconds, which is adequate for pedestrian walking. Along with the measurement of RSS our central console will also record where the RSS reading coming from. We also record a timestamp for every measurement. As above, the result for each beacon's RSS reading would be a tuple like (1):

$$
\text { (Beacon's MAC, RSS, timestamp) }
$$

First element of the tuple is the MAC address of the beacon device. Second is the RSS indicator in decibel. Finally the third is the timestamp represent when this tuple was captured. Assuming that there are $n$ beacons planted in the building. Then we can get $n$ triple tuples as (2):

$$
\left(X_{1}, X_{2}, X_{3}, \ldots, X_{n}\right)
$$

$X_{n}$ is the tuple recorded from the $n$th beacon device, and it conforms to the format of (1).

\section{Mathematical Basics and Filter Design}

We employ classic finger-print approach to start and continue the recursion. During the recursion, we use a Bayesian condition matrix to update the distribution. Our condition matrix is constructed automatically from the building layout.

According to Bayes filter we represent the state at time $t$ by $x_{t}$, represent the uncertainty by a probability distribution called belief $\operatorname{Bel}\left(x_{t}\right)$. Assuming the observation is $z_{1}, z_{2}, \ldots, z_{t-1}$, then the belief at time $t$ can be defined as (3):

$$
\operatorname{Bel}\left(x_{t}\right)=p\left(x_{t} \mid z_{1}, z_{2}, \ldots, z_{t-1}\right)
$$

Based on this assumption, we implement our filter in two procedures and invoke them recursively. The first is update and the second is prediction. And their relationship is in Fig.1. As the illustration shows, update brings noise to the distribution 
while prediction filters the noise. And deviations caused by prediction will be corrected by update.

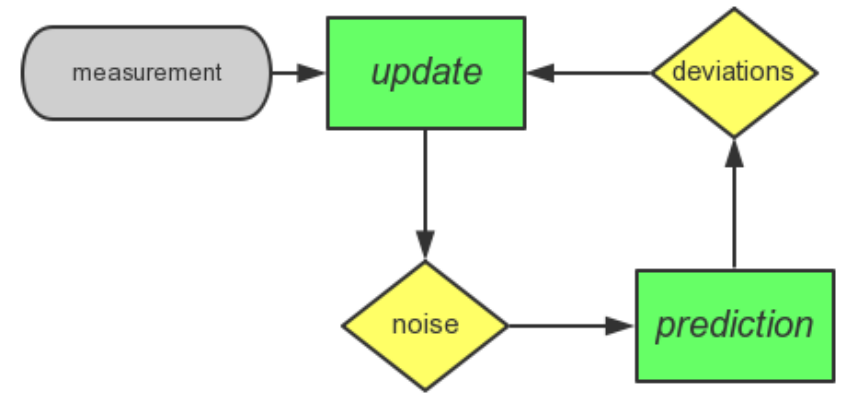

Fig.1. Filter Procedure

If the building has a regular layout and obstacles are relative static, we can estimate the approximation benchmark RSS according to following formula like (4):

$$
P_{L}(d)=P_{L}\left(d_{0}\right)+10 n \log \left(\frac{d}{d_{0}}\right)+X_{\sigma L}
$$

where $P_{L}(d)$ is the received signal strength in a distance d, $P_{L}\left(d_{0}\right)$ is also signal strength but in a reference distance $d_{0}, n$ is the path loss exponent and $X_{\sigma L}$ represents the noise using a random variable with normal distribution, zero mean and standard deviation $\sigma L$. There are many variants of this formula and their usage is the same: measuring several standard variables then concluding the whole hologram of signal distribution. We will concentrate our enthusiasm on the custom design of the filter, so we are going to use empirical data instead of calculation in section IV. We use the calculation or empirical data from above to initialize a map for latter comparison. For example, if we have collected $m$ finger-prints for all the positions, the map would be a matrix like (5) including vectors formatted in (2):

$$
\left(\overrightarrow{V_{1}}, \overrightarrow{V_{2}}, \ldots, \overrightarrow{V_{m}}\right)
$$

Before update we can initialize our probability distribution according to the real world information, or we just initialize them by average. For the vectors representing $m$ positions in (5), we can initialize the distribution with probabilities all equal to $1 / \mathrm{m}$. After that, we start update. The only information resource of update is the measurement of RSS and its comparison with former history record.

Imagining that we have 5 pre-defined position, before the first update, we initialize the probability distribution by average which is shown in Fig.2a. Once the measurements imply a confidence by probability for each position, the original distribution will be updated. The mathematical procedure is quite easy. First multiply each position's belief by each one's confidence. Then normalize the whole distribution. For example, if we are $80 \%$ sure that target is at position 3 and $20 \%$ sure that it's at other positions, then the distribution will be updated like Fig.2b.

After update we can start prediction. Prediction needs Bayes conditions to adjust the original distribution. We employ a statetransition matrix to represent the conditions. Assuming that the

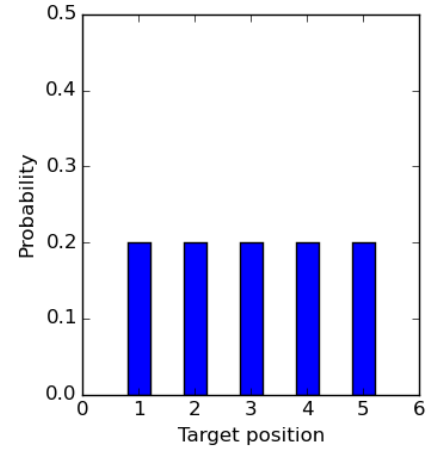

(a)

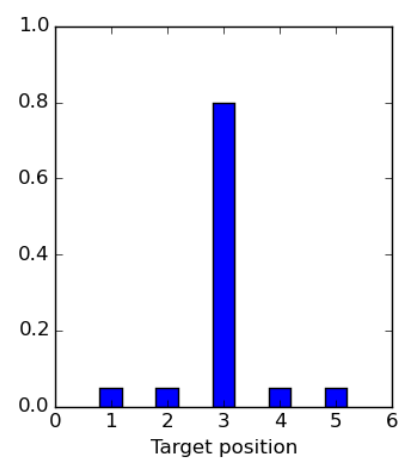

(b)
Fig.2. Distribution after update

five positions of Fig.2 is modeled into a topological graph. We adopt topological searching algorithm to determine the connections. After that we set our assumptions according to the real conditions. Simple assumptions are: (1) Target can only go to connected points or just stay where it is. (2) The possibilities of target going or staying are the same. (3) The possibilities of target going to connected point are divided equally.

These assumptions can be applied to the topological graph and generate a symmetric matrix shown in (6) which represents the Bayes conditions. The data in this matrix are supposed to be more advance than the ones based on those three simple assumptions. In section IV we will use a matrix based on Gaussian distribution.

$$
\left[\begin{array}{ccccc}
0.5 & 0 & 0 & 0.5 & 0 \\
0 & 0.5 & 0.25 & 0 & 0.16 \\
0.5 & 0.25 & 0.5 & 0 & 0.16 \\
0.5 & 0 & 0 & 0.5 & 0.16 \\
0 & 0.16 & 0.16 & 0.16 & 0.5
\end{array}\right]
$$

Finally, we predict the probability distribution of next state. Prediction procedure synthesizes the original distribution with the static symmetric matrix by convolution. Take the first position as example. Multiply each possibilities of Fig.2b with each possibilities of the first column of (6) and sum them together to be the prediction possibility of certain position. Prediction is over for now. After this, the recursion will go on automatically. The series of measurement at time $t$ constructs the conditions of (3). Update procedure corrects probability distribution like Fig.2a into Fig. $2 b$ which actually records the history of $z_{t-1}, z_{t}$. Then prediction procedure gives the next state of distribution according to previous history and the statetransition matrix.

\section{EXPERIMENT EVALUATION}

Experiment is divided into two parts. The first part is merely finger-print approach and its results. The second part is experimental results of our filter. Evaluation and comparison are shown in Fig.3 and Fig.4 to illustrate the advantage of our implementation.

The simulation ground is along a hallway with a corner. Its layout is shown in Fig.3. And the location estimation of each step is calculated by the centroid weighted algorithm $k-N N$. The 
weights are determined by the possibility of target being at each point for current step. According to this rule of calculation, Fig.3 shows a comparison between an estimated path of merely fingerprint approach and an estimated path of Bayes filter approach.

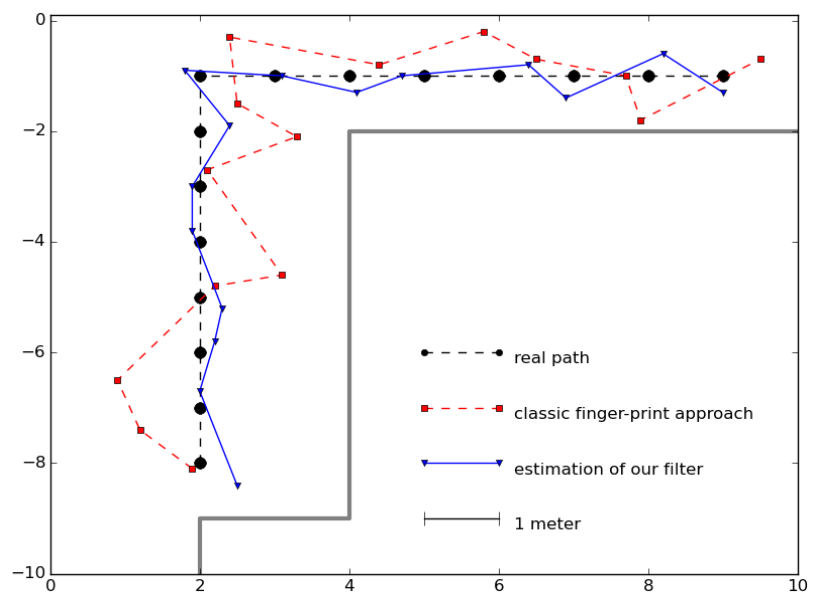

Fig.3. Real path and trajectory estimation

In Fig.3, the object's real trajectory is along the round dots. The estimation based on RSS finger-print without filter is shown along the square dots while the estimation with Bayes filter is shown along the triangular dots. Since target moves in irregular shuttle directions, the deviations of each step are not clear. We calculate the deviations for all steps and show them in Fig.4. The simulation results show the Bayes filter can give better estimation with smaller error and covariance.

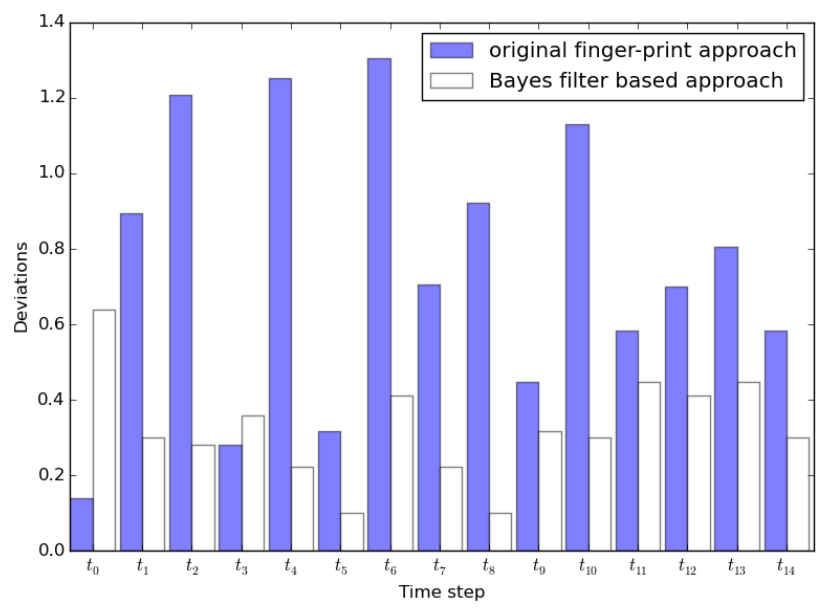

Fig.4. Deviations statistics

\section{CONCLUSION AND FUTURE WORK}

Localization of indoor environment has various appealing applications and a bright future. However, its infrastructure still need to depend on existing technologies and available devices. For indoor localization, the most widely used are Wi-Fi, Bluetooth, RFID, etc. This paper proposed a system implementation of indoor localization based on BLE which supports lots of off-the-shelf devices. It utilized Bayes filter to optimize localization of indoor object. The implementation section describes how to employ building layouts and object movement information as Bayes conditions. The empirical evaluations show improvement in the accuracy of estimation.

The recursive procedure mentioned in section III conforms to Hidden Markov Model which implies our state transition matrix can be established based on any other information besides building layouts. However, collecting more information as Bayes conditions means more fundamental training work which may bring much more cost of deployment and maintenance. In our implementation the object's movement is predicted without much reasoning. We speculate that object can go to all the connected position with equal possibility. Actually, we can use the sensors from mobile device to gain more information about the object's movement. For example, by the build-in gyroscope and accelerometer. The future work can be carried out by the combination of better scene profiling methods and our system. In this way, the state-transition matrix can be established more efficiently and the estimation of position will also be narrowed down.

\section{REFERENCES}

[1] Paramvir Bahl and Venkata N. Padmanabhan. "RADAR: An in-building RF-based user location and tracking system." INFOCOM 2000. Nineteenth Annual Joint Conference of the IEEE Computer and Communications Societies. Proceedings. IEEE. Vol. 2. Ieee, 2000.

[2] Roy Want, Andy Hopper, Veronica Falcão and Jonathan Gibbons. "The active badge location system." Acm Transactions on Information Systems 10.1(1992):91-102.

[3] ANDY HARTER, ANDY HOPPER, PETE STEGGLES, ANDY WARD and PAUL WEBSTER. "The Anatomy of a Context-Aware Application." Wireless Networks 8.2-3(2002):187--197.

[4] Teng Li, Anthony Ekpenyong and Yih-Fang Huang. "Indoor positioning techniques based on wireless LAN." LAN, First IEEE International Conference on Wireless Broadband and Ultra Wideband Communications. 2006.

[5] Motley, A. J. and J. M. P. Keenan. "Personal communication radio coverage in buildings at $900 \mathrm{MHz}$ and $1700 \mathrm{MHz}$." Electronics Letters 24.12 (1988): 763-764.

[6] ANDY HARTER, ANDY HOPPER, PETE STEGGLES, ANDY WARD and PAUL WEBSTER. "The Anatomy of a Context-Aware Application." Wireless Networks 8.2-3(2002):187--197.

[7] Dragos Niculescu and Badri Nath. "VOR base stations for indoor 802.11 positioning." Proceedings of the 10th annual international conference on Mobile computing and networking ACM, 2004.

[8] Xinrong Li and Kaveh Pahlavan. "Super-resolution TOA estimation with diversity for indoor geolocation." Wireless Communications, IEEE Transactions on 3.1 (2004): 224-234.

[9] Li, Teng, Anthony Ekpenyong, and Yih-Fang Huang. "A location system using asynchronous distributed sensors." INFOCOM 2004. Twenty-third AnnualJoint Conference of the IEEE Computer and Communications Societies. Vol. 1. IEEE, 2004.

[10] Teng Li, Anthony Ekpenyong and Yih-Fang Huang. "Indoor positioning techniques based on wireless LAN." LAN, First IEEE International Conference on Wireless Broadband and Ultra Wideband Communications. 2006 